\title{
Biomarkers under investigation for neoadjuvant immune checkpoint inhibitors in non-small cell lung cancer
}

\author{
Yasemin Söyler^, Ülkü Yılmaz^^ \\ Department of Chest Diseases, Health Sciences University Atatürk Chest Diseases and Chest Surgery Health Research and Application Center, \\ Ankara, Turkey \\ Contributions: (I) Conception and design: Both authors; (II) Administrative support: U Y1lmaz; (III) Provision of study materials or patients: Both \\ authors; (IV) Collection and assembly of data: Y Söyler; (V) Data analysis and interpretation: Both authors; (VI) Manuscript writing: Both authors; \\ (VII) Final approval of manuscript: Both authors. \\ Correspondence to: Yasemin Söyler, MD. Pınarbası Mah. Sanatoryum Caddesi, 06280, Kecioren, Ankara, Turkey. Email: yaseminsoylerdr@gmail.com.
}

\begin{abstract}
The 5-year relative survival of non-small cell lung cancer (NSCLC) is still low even in patients with resectable disease due to local or distant recurrences after surgery. Novel treatment strategies are essential to reduce the risk of postoperative recurrence and provide an improvement in survival rates. Although neoadjuvant immune checkpoint inhibitors (ICIs) are not included in the treatment protocols yet, investigations are still ongoing. However, one of the major issues encountered in this regard is the ideal patient selection. Furthermore, ICIs have several limitations such as serious side effects, high costs and varying response rates among patients. Thus, the development of accurate biomarkers is necessary for ideal patient selection, risk stratification and prediction of treatment response in patients. Biomarkers under investigation for neoadjuvant ICIs in NSCLC are being evaluated in four major categories: tumour cellassociated biomarkers, tumour microenvironment-related biomarkers, liquid biopsy-related biomarkers and host-related markers. In addition, ${ }^{18}$ F-FDG PET-CT parameters may be used in this regard. Emerging results from clinical trials suggest that these biomarkers may play important roles in the management of NSCLC in the near future. Nevertheless, there are still many unknowns about mechanisms of biomarkers. Some challenges, such as clinical adaptability, validation and feasibility, also exist. It should be noted that, there are no currently standardized biomarkers to select the ideal patient. Further studies are therefore required to identify reliable biomarkers in the prediction of response and outcome of ICIs.
\end{abstract}

Keywords: Biomarkers; neoadjuvant immune checkpoint inhibitors (ICIs); non-small cell lung cancer (NSCLC); programmed death ligand 1 (PD-L1) expression; tumour mutation burden (TMB)

Received: 09 August 2021; Accepted: 09 June 2022; Published: 01 December 2022.

doi: $10.21037 /$ asj-21-75

View this article at: https://dx.doi.org/10.21037/asj-21-75

\section{Introduction}

Non-small cell lung cancer (NSCLC) is the most frequent subtype of lung cancer which is one of the most lethal cancers worldwide. Although its incidence rates and ageadjusted death rates have been falling each year, the 5-year relative survival is still low even in patients with resectable disease due to local or distant recurrences after surgery (ranging from 25\% to 70\%) (1-3). Neoadjuvant and adjuvant treatment strategies such as chemotherapy, targeted therapy and immunotherapy have therefore received much attention in the last years to reduce the risk of postoperative recurrence of resectable NSCLC and provide an improvement in survival rates (2). The use of cancer immunotherapy, especially immune checkpoint inhibitors (ICIs), has ushered

^ ORCID: Yasemin Söyler, 0000-0002-0507-0767; Ülkü Yılmaz, 0000-0003-1493-8385. 
Table 1 Clinical trials of neoadjuvant therapy with ICIs for resectable NSCLC

\begin{tabular}{|c|c|c|c|c|c|c|}
\hline $\begin{array}{l}\text { Trial name/registration } \\
\text { number }\end{array}$ & Phase & Stage & $\begin{array}{l}\text { Actual/estimated } \\
\text { enrollment }(n)\end{array}$ & Treatment & Primary endpoint & $\begin{array}{l}\text { Trial completion } \\
\text { date/estimated trial } \\
\text { completion date }\end{array}$ \\
\hline ChiCTR-OIC-17013726 & $1 \mathrm{~B}$ & IB-IIIA & 22 & Sintilimab & $\begin{array}{l}\text { Drug-related adverse } \\
\text { event, surgery } \\
\text { complications, no- } \\
\text { delay surgery rate }\end{array}$ & 2019 \\
\hline $\begin{array}{l}\text { Checkmate 159/ } \\
\text { NCT02259621 }\end{array}$ & 2 & IB-IIIA & 45 & Nivolumab & Safety and feasibility & 2023 \\
\hline LCMC3/NCT02927301 & 2 & IB-IIIB & 181 & Atezolizumab & MPR & 2020 \\
\hline NEOSTAR/NCT03158129 & 2 & I-IIIA & 88 & Nivolumab & MPR & 2021 \\
\hline $\begin{array}{l}\text { Columbia University/ } \\
\text { NCT02716038 }\end{array}$ & 2 & IB-IIIA & 30 & Atezolizumab & MPR & 2020 \\
\hline NADIM II NCT03838159 & 2 & IIIA/IIIB & 90 & Nivolumab & $\mathrm{pCR}$ & 2022 \\
\hline TOP 1501/NCT02818920 & 2 & IB-IIIA & 35 & Pembrolizumab & Surgical feasibility & 2026 \\
\hline IONESCO/NCT03030131 & 2 & IB-II & 81 & Durvalumab & R0 resection & 2019 \\
\hline PRICNEPS/NCT0299457 & 2 & IB-IIIA & 60 & Atezolizumab & Toxity & 2020 \\
\hline \multirow[t]{2}{*}{ NeoCOAST/NCT03794544 } & 2 & I-IIIA & 160 & Durvalumab & MPR & 2021 \\
\hline & & & & Durvalumab + Oleclumab & & \\
\hline $\begin{array}{l}\text { Checkmate 816/ } \\
\text { NCT02998528 }\end{array}$ & & & & Nivolumab + ipilimumab & & \\
\hline Keynote-671/NCT03425643 & 3 & II-IIIB & 786 & Pembrolizumab & EFS, OS & 2024 \\
\hline AEGEAN/NCT03800134 & 3 & IIA-IIIB & 300 & Durvalumab & MPR & 2024 \\
\hline
\end{tabular}

ICls, immune checkpoint inhibitors; NSCLC, non-small cell lung cancer; MPR, major pathological response; PFS, progression-free survival; pCR, pathological complete response; EFS, events-free survival; OS, overall survival.

a new era for oncology and led to great hope. Although ICIs are not included in the neoadjuvant treatment protocols yet, investigations on this subject are still ongoing. Currently, there are various ongoing and completed clinical trials (phases I, II and III) underway to assess the role of neoadjuvant ICIbased treatments in patients with resectable NSCLC (Table 1). However, one of the major issues encountered in this regard is the ideal patient selection. Furthermore, ICIs have several limitations such as serious side effects, high costs and varying response rates among patients (4). Thus, the development of biomarkers is necessary for ideal patient selection, risk stratification and prediction of treatment response in patients with early stage lung cancer. They are ideal settings for exploring biomarkers due to emerge novel pathways 


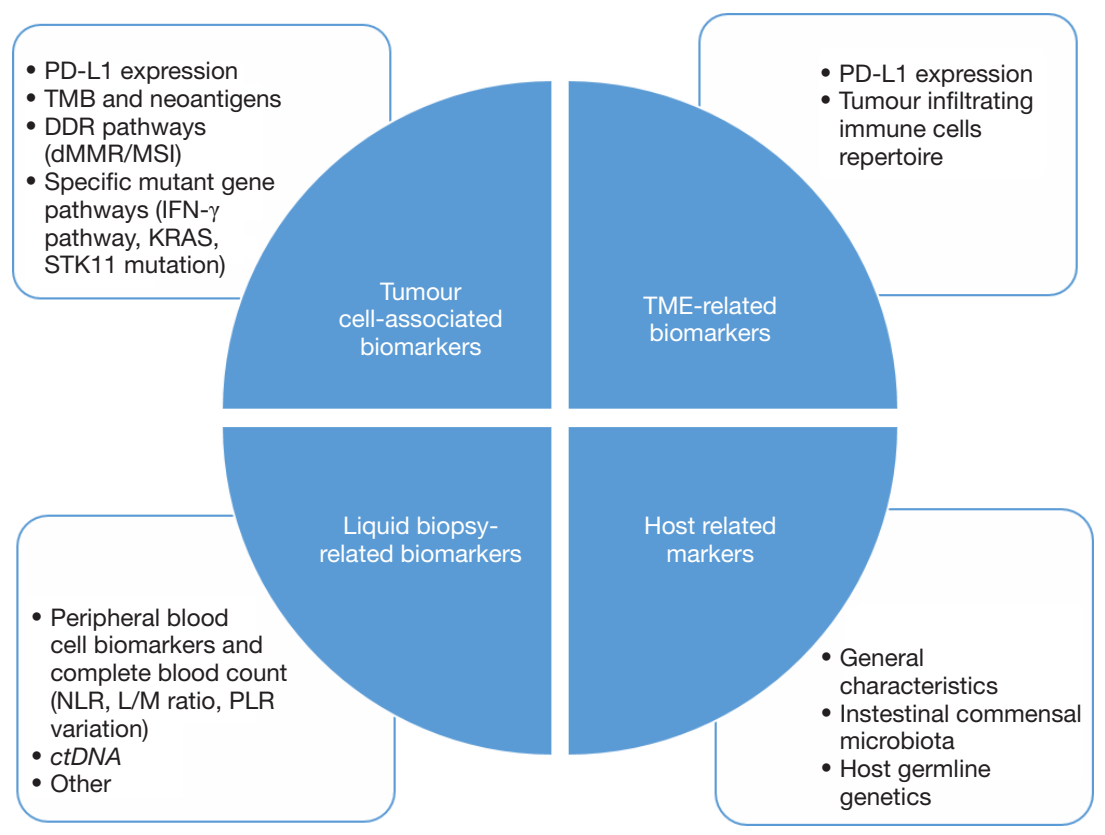

Figure 1 Biomarkers under investigation for neoadjuvant immune checkpoint inhibitors in non-small cell lung cancer. PD-L1, programmed death ligand 1; TMB, tumour mutation burden; DDR, DNA damage response; dMMR, DNA mismatch repair deficiency; MSI, microsatellite instability; TME, tumour microenvironment; NLR, neutrophil to lymphocyte ratio; L/M ratio, myeloid to lymphoid ratio; PLR, platelet to lymphocyte ratio; ctDNA, circulating tumour DNA.

and molecules during studies about neoadjuvant ICIs. As results of these studies, biomarkers under investigation for neoadjuvant ICIs in NSCLC are being evaluated in four major categories (Figure 1): tumour cell-associated biomarkers, tumour microenvironment-related biomarkers, liquid biopsy-related biomarkers and host-related markers $(2,5)$. In addition, ${ }^{18} \mathrm{~F}$-FDG PET-CT parameters may be used as clinical indicator of tumour immunometabolic phenotypes in patients with resectable NSCLC (6).

\section{Tumour cell-associated biomarkers}

Two biomarkers mostly studied in the context of cancer immunotherapy are programmed death ligand 1 (PD-L1) expression and tumour mutation burden (TMB). Tumour cell-associated biomarkers also include DNA damage response (DDR) pathways, specific mutant gene pathways (e.g., IFN- $\gamma$ pathway, KRAS and STK11 mutation), and neoantigen load (2).

\section{PD-L1 expression}

As PD-L1 is expressed on the surface of tumour cells to inhibit $\mathrm{T}$ cells and survive their cytotoxic activities, degree of immunohistochemical measurement of PD-L1 in biopsy materials is being investigated as a biomarker of responses to ICIs (3). However, the role of PD-L1 is still under debate due to the disparities between previous studies (3,7-9). For instance, pretreatment PD-L1 expression was higher in responders than in non-responders and was positively correlated with major pathological response (MPR) in the NEOSTAR study, but it was not correlated with MPR in the LCMC3 study $(10,11)$. The CheckMate-159 study also found no association between MPR and PD-L1 expression (12). The disparities may be explained with PD-L1 expression pattern that is temporally and spatially heterogeneous, and also dynamic. This indicates that it may vary in different anatomical regions and during the clinical course $(13,14)$. It may also be affected by histological type of tumour, tissue availability prior to therapy, length of neoadjuvant ICI therapy or chemotherapy/radiation $(3,8)$. Intratumour heterogeneity in NSCLC may be another explanation (15). Furthermore, there is a lack of standardization across PD-L1 platforms $(8,13,16)$. Taken together, the results on using PD-L1 expression as a biomarker need confirmation with further studies. 


\section{TMB and neoantigens}

TMB can be defined as the total number of nonsynonymous single-nucleotide mutations, insertion or deletion variants that gives rise to tumour-specific neoantigens which in turn can activate the host $\mathrm{T}$ cell response against tumour $(3,17)$. Neoantigens are foreign proteins which form on cancer cells when certain mutations occur in tumour DNA. They help the immune system to recognize the affected cancer cell and thus play an important role in immune escape, immunoediting, and sensitivity to ICIs (18). Theoretically, a higher TMB increases the probability of tumour neoantigen production and therefore, the likelihood of immune recognition and tumour cell killing (19).

TMB may vary between different tumours and one of the highest levels of TMB was documented in NSCLC (20). In the CheckMate-159 study with 21 patients with earlystage NSCLC treated with neoadjuvant nivolumab, the achievement of a MPR was highly associated with an increased TMB (12). In another study with patients with resected NSCLC, high nonsynonymous TMB was found to be associated with a better prognosis (21). Although previous studies showed positive results, the LCMC3 study found no correlation between TMB and MPR (11). Additionally, TMB was not found to be associated with survival in the NADIM study (46 patients with stage IIIA NSCLC) (22). TMB has also some other limitations. For instance, there may be a high degree of variation in TMB across patients within NSCLCs because of its association with smoking $(16,21)$. A previous study, which analyzed the most important confounders of panel sequencing based measurement of TMB (psTMB) (panel size, germline filtering, biological and technical variance), showed that the limited panel size represented the largest contributor to total psTMB variance (23). It was also shown that in addition to technical aspects such as germline filtering, the tumour content and spatially divergent mutational profiles within a tumour were relevant factors influencing TMB estimation, revealing limitations of singlesample-based TMB estimations (24). Furthermore, the best measurement method for TMB is whole exome sequencing (WES), but it is not yet routinely used as a clinical tool for predicting response to ICIs due to its high cost and long turn-around time (16).

The influence of neoantigens on antitumor immunity in early-stage NSCLC has also been explored. A high neoantigen burden, identified as the upper quartile of neoantigen load, was associated with significantly longer OS. In addition, combining high mutational load and low intratumoral neoantigen heterogeneity $(<1 \%)$ was significantly associated with OS and longer lasting clinical benefit than either variable alone (25). These results support studies exploring neoantigens as potential biomarkers of tumour response to neoadjuvant ICIs in patients with resectable NSCLC (3).

\section{DDR patbways [DNA mismatch repair deficiency (dMMR)/microsatellite instability (MSI)]}

Five clinical trials (Keynote-016, 164, 012, 028, 158) with various types of tumours reported that patients with dMMR/ MSI-H have durable responses to pembrolizumab (13). However, a study in patients with surgically resected lung adenocarcinoma reported that MSI was a rare event and MMR deficiency status cannot be used as a biomarker for ICI treatment in lung adenocarcinoma (26).

\section{Tumour microenvironment-related biomarkers}

Analyzes of the tumour microenvironment have revealed that it is an important factor in cancer progression and metastasis (27). PD-L1 expression, and tumour-infiltrating immune cells, including immune cells with specific phenotypes (e.g., CD $39^{+}, \mathrm{CD}^{+} \mathrm{T}, \mathrm{CD} 4^{+} \mathrm{T}$ cells, FoxP3 ${ }^{+}$ $\mathrm{T}$ cells, NKp $46^{+}$cells), diversity of immune repertoires [e.g., richness and clonality of tumour-infiltrating lymphocytes (TILs), T cell receptor (TCR) repertoire], and immune status score have been explored as tumour microenvironment-related biomarkers in lung cancer (2).

\section{Tumour infiltrating immune cells repertoire}

Basically, tumour infiltrating immune cells include both mononuclear and polymorphonuclear immune cells. One of the major components of them are TILs which can be used as biomarkers in predicting the efficacy and outcome of treatment in a number of solid tumours, including lung cancer. Higher infiltration of TILs was found to be associated with improved survival in NSCLC $(28,29)$. Several studies have also supported these results by demonstrating a high correlation between TILs density and both disease outcome and treatment response in lung cancer. In the NADIM study the found an association between TIL levels in post-treatment surgical specimens and progression-free survival (PFS). They reported that patients with low stromal levels of $\mathrm{CD}^{+} \mathrm{CD}^{+}$cells, $\mathrm{CD} 3^{+}$ PD-L1 cells, or with low tumour levels of $\mathrm{CD} 3^{+} \mathrm{CD} 8^{+}$ 
PD-L $1^{+}$cells or $\mathrm{CD}^{+}{ }^{+} \mathrm{PD}-\mathrm{L}^{+}{ }^{+}$cells showed all a $100 \%$ PFS at both 18 and 24 months (22). In another study with stage I NSCLC patients reported that a higher proportion of tumour $\mathrm{T}_{\text {reg }}$ cells (FoxP3 ${ }^{+} \mathrm{TILs}$ ) relative to TIL had a significantly higher risk of recurrence (30). Regarding the potential important of immune cell subsets, a previous study suggested that B cells and total TILs could be complementary predictors of ICI benefit in NSCLC. Because they found that a higher proportion of B cells, CD45+ cells and total TILs was associated with prolonged PFS after ICI treatment. The feasibility of TIL/TMEprofiling in the routine setting using mRNA assays was also demonstrated in this study (31). In lung cancer, however, there is still a lack of a standardized guidelines for TIL scoring/use (32).

TCRs are used to recognise neoantigen peptides, identify $T$ cell clones, their frequencies, and the existence of antigenic responses within a repertoire $(3,33)$. The sum of all TCRs by the T cells of one individual is named as TCR repertoire. TCR repertoire features, including the frequency of $\mathrm{T}$ cells within a tumour (density), the diversity of $\mathrm{T}$ cells (richness), and the tumour reactive $\mathrm{T}$ cell clonal expansion (clonality), may be potential biomarkers in lung cancer. A previous study with 236 early-stage, treatment-naïve NSCLC patients reported that higher $\mathrm{T}$ cell repertoire homology between the tumour and uninvolved tumour-adjacent lung was associated with inferior survival (33). However, less information is known about the attributes of TCR repertoire as biomarkers of response to neoadjuvant ICIs in NSCLC patients and large-scale studies are needed $(3,33)$.

Decision on the treatment, and prognostic prediction are based on the American Joint Committee on Cancer TNM staging system and the likelihood of tumour recurrence varies at each tumour stage. However, clinical outcomes of patients within the same tumour stage can also vary. Using the TNM staging system alone may not predict response to therapy and may be insufficient for precise assessment of patient survival (27). Therefore, researchers developed the immunoscore model and immunoscore-clinical prognostic signature (ICPS). They reported that high immunoscore was positively correlated with TMB and associated with worse prognosis, suggesting that immunoscore and ICPS can be used for prediction of survival in early-stage lung adenocarcinoma (27).

\section{Liquid biopsy-related biomarkers}

Liquid biopsy-related biomarkers include peripheral blood cells (e.g., CD45RO ${ }^{+} / \mathrm{CD}^{+} \mathrm{T}$ cells, $\mathrm{CD}^{+} \mathrm{ICOS}^{+} \mathrm{T}$ cells, circulating tumour cells), circulating tumour DNA (ctDNA), and other circulating molecular biomarkers (e.g., exosomes, cytokines, and inflammatory factors) (2). They have significant advantages of being non-invasive as well as timeand cost-efficient. Thus, they may frequently be preferred for monitoring response to neoadjuvant or adjuvant immunotherapy in the future.

\section{Peripheral blood cell biomarkers and complete blood count}

In the LCMC3 study, the biomarker analysis based on paired peripheral blood demonstrated a lower baseline levels of $\mathrm{T}$ cells $\left(\mathrm{G} / \mathrm{D}^{+} \mathrm{CD} 3^{+} \mathrm{CD} 8^{+} \mathrm{CD} 56^{+} \mathrm{CD} 16^{+}\right)$and natural killer cells $\left(\mathrm{CD} 16^{+} \mathrm{CD} 56^{+} \mathrm{CD} 244^{+} \mathrm{CD} 314^{+} \mathrm{CD} 161^{+}\right)$in patients who achieved MPR compared with patients without MPR. After neoadjuvant treatment, the absolute cell counts of late activated $\mathrm{CD} 4^{+}$and $\mathrm{CD} 8^{+} \mathrm{T}$ cells decreased in patients who achieved MPR. The results from this study revealed the role of ICIs in preoperative activation of tumour-specific immune killing $(2,34)$. In another study, the association between higher $\mathrm{T}$ cell density in the peripheral blood and improved outcome/longer overall survival (OS) after surgery in NSCLC was revealed and the authors suggested that the peripheral $\mathrm{T}$ cell repertoire may be reflective of increased systemic immunity (33). In the CheckMate-159 study, anti-PD-1 therapy increased the number of neoantigenspecific $\mathrm{T}$ cell clones in tumour and peripheral blood in resectable NSCLC (12). Since most of the mentioned biomarkers are expensive, time consuming and experience dependent, the need for more easily accessible biomarkers in daily clinical practice, such as complete blood cell counts, has arisen. A cohort of 1,524 patients with surgically resected NSCLC from the ICON study identified an association between increased circulating neutrophil expansion with disease burden and worse postoperative OS (18). In many solid tumours and advanced NSCLC, high neutrophil-to-lymphocyte ratio (NLR) and platelets-to-lymphocytes ratio (PLR) have been reported to be associated with an adverse OS (35). In the NADIM study, post-treatment peripheral NLR, myeloidto-lymphoid ratio (MLR), and PLR were decreased and a greater decrease in the post-treatment PLR was associated with pathologic complete response (36).

These results suggest that peripheral blood can be used as a valuable source to detect potential biomarkers for ICIs. However, further investigations are required to use these tests as biomarkers in routine practice in patients receiving 
neoadjuvant immunotherapy since its effectiveness has not been validated in prospective studies (13).

\section{$c t D N A$}

ctDNA, single- or double-stranded DNA derived from tumour cells, contains the mutations of the original tumour (18). Thus, the detection of ctDNA can obtain tumour genomic information related to the response to ICIs and can be used as a biomarker (13). For instance, the Checkmate-816 study, which is the first phase III trial to show a benefit for neoadjuvant ICI (nivolumab) plus platinum-doublet chemotherapy for resectable NSCLC, demonstrated that patients treated with nivolumab plus chemotherapy were more likely to have ctDNA clearance and appeared to be more likely to have a pathological complete response at the time of surgery (37). The CheckMate-159 study also reported that the clearance of detectable ctDNA from blood prior to surgery was detected in all patients who achieved a reduction $\geq 30 \%$ in viable tumour in response to nivolumab (12). Another study demonstrated the association between the detection of ctDNA in peripheral blood as early as 4 weeks following tumour resection and elevated risk of disease recurrence in NSCLC. They also suggested that ctDNA detection can be used for assessment of tumour relapse in patients who have undergone curative surgery (38). Although the sensitivity or specificity has yet to be improved, the results from previous studies are promising.

\section{Other circulating molecular biomarkers}

Exosomes which are small sized extracellular vesicles, can transport functional biomolecules, such as small RNAs, DNAs, proteins and lipids. Due to their presence and stability in most biological fluids and their contents reflecting abnormalities in the parent cells, they may be potential liquid biopsy biomarkers for various diseases, including lung cancer $(39,40)$.

Soluble proteins (e.g., sCD163, sNKG2DLs), cytokines and inflammatory factors (e.g., tumour necrosis factor- $\alpha$, interleukin-6, C-reactive protein) are also being investigated as potential predictive biomarkers for ICIs efficacy (13).

\section{Host-related markers}

General characteristics (gender, age, performance status and body fat distribution), intestinal commensal microbiota, and host germline genetics (human leukocyte antigen diversity and other specific mutations) can be evaluated as biomarkers in various cancer types. Further studies are required to explore the effect mechanisms of general characteristics and the impact of host immune gene variation on ICIs efficacy (13).

\section{${ }^{18}$ F-FDG PET-CT parameters}

${ }^{18}$ F-FDG PET-CT plays an important role in the diagnosis, staging and follow-up of lung cancer and parameters such as maximum standardized uptake values $\left(\mathrm{SUV}_{\max }\right)$ may provide valuable information for efficacy evaluation in neoadjuvant treatment in NSCLC. In a study with 172 patients with resected NSCLC demonstrated that increased $\mathrm{SUV}_{\max }$ was associated with multiple immune factors characteristic of an immunosuppressive and poorly immune infiltrated tumour microenvironment, including elevated PD-L1 expression, reduced $\mathrm{CD}_{5} 7^{+}$cell density, and increased $\mathrm{T}$ cell exhaustion gene signature (6). The results of another study suggest that the decrease of $\mathrm{SUV}_{\max }$ values after neoadjuvant sintilimab could be predictive of pathologic response to neoadjuvant PD-1 blockade (41).

\section{Conclusions}

Emerging results suggest that biomarkers for neoadjuvant ICIs may play important roles in the management of NSCLC in the near future. Nevertheless, there are still many unknowns about mechanisms of biomarkers. Some challenges, such as clinical adaptability, validation and feasibility, also exist. It should be noted that, there are currently no standardized biomarkers to select the ideal patient. Further studies are therefore required to identify reliable biomarkers in the prediction of response and outcome of ICIs.

\section{Acknowledgments}

Funding: None.

\section{Footnote}

Provenance and Peer Review: This article was commissioned by the Guest Editors (Dragana Jovanovic and Semra Bilaceroglu) for the series "Impact of Novel Neoadjuvant Treatment on Surgery Outcomes in Lung Cancer" published in AME Surgical fournal. The article has undergone external peer review. 
Peer Review File: Available at https://asj.amegroups.com/ article/view/10.21037/asj-21-75/prf

Conflicts of Interest: Both authors have completed the ICMJE uniform disclosure form (available at https://asj. amegroups.com/article/view/10.21037/asj-21-75/coif). The series "Impact of Novel Neoadjuvant Treatment on Surgery Outcomes in Lung Cancer" was commissioned by the editorial office without any funding or sponsorship. The authors have no other conflicts of interest to declare.

Ethical Statement: The authors are accountable for all aspects of the work in ensuring that questions related to the accuracy or integrity of any part of the work are appropriately investigated and resolved.

Open Access Statement: This is an Open Access article distributed in accordance with the Creative Commons Attribution-NonCommercial-NoDerivs 4.0 International License (CC BY-NC-ND 4.0), which permits the noncommercial replication and distribution of the article with the strict proviso that no changes or edits are made and the original work is properly cited (including links to both the formal publication through the relevant DOI and the license). See: https://creativecommons.org/licenses/by-nc-nd/4.0/.

\section{References}

1. Howlader N, Noone A, Krapcho M, et al. SEER Cancer Statistics Review, 1975-2017. National Cancer Institute, Bethesda, MD. November 2019 SEER data submission, posted to the SEER web site, April 2020. Available online: https://seer.cancer.gov/csr/1975_2017/

2. Bai R, Li L, Chen X, et al. Neoadjuvant and Adjuvant Immunotherapy: Opening New Horizons for Patients With Early-Stage Non-small Cell Lung Cancer. Front Oncol 2020;10:575472.

3. Pradhan M, Chocry M, Gibbons DL, et al. Emerging biomarkers for neoadjuvant immune checkpoint inhibitors in operable non-small cell lung cancer. Transl Lung Cancer Res 2021;10:590-606.

4. Music M, Prassas I, Diamandis EP. Optimizing cancer immunotherapy: Is it time for personalized predictive biomarkers? Crit Rev Clin Lab Sci 2018;55:466-79.

5. Mielgo-Rubio X, Calvo V, Luna J, et al. Immunotherapy Moves to the Early-Stage Setting in Non-Small Cell Lung Cancer: Emerging Evidence and the Role of Biomarkers. Cancers (Basel) 2020;12:3459.
6. Mitchell KG, Amini B, Wang Y, et al. 18F-fluorodeoxyglucose positron emission tomography correlates with tumor immunometabolic phenotypes in resected lung cancer. Cancer Immunol Immunother 2020;69:1519-34.

7. Bocanegra A, Fernandez-Hinojal G, Zuazo-Ibarra M, et al. PD-L1 Expression in Systemic Immune Cell Populations as a Potential Predictive Biomarker of Responses to PDL1/PD-1 Blockade Therapy in Lung Cancer. Int J Mol Sci 2019;20:1631.

8. Tsoukalas N, Kiakou M, Tsapakidis K, et al. PD-1 and PD-L1 as immunotherapy targets and biomarkers in nonsmall cell lung cancer. J BUON 2019;24:883-8.

9. Takada K, Okamoto T, Shoji F, et al. Clinical Significance of PD-L1 Protein Expression in Surgically Resected Primary Lung Adenocarcinoma. J Thorac Oncol 2016;11:1879-90.

10. Cascone T, William W, Weissferdt A, et al. Neoadjuvant nivolumab $(\mathrm{N})$ or nivolumab plus ipilimumab (NI) for resectable non-small cell lung cancer (NSCLC): Clinical and correlative results from the NEOSTAR study. J Clin Oncol 2019;37:8504.

11. Kwiatkowski D, Rusch V, Chaft J, et al. Neoadjuvant atezolizumab in resectable non-small cell lung cancer (NSCLC): Interim analysis and biomarker data from a multicenter study (LCMC3). J Clin Oncol 2019;37:abstr 8503.

12. Forde PM, Chaft JE, Smith KN, et al. Neoadjuvant PD-1 Blockade in Resectable Lung Cancer. N Engl J Med 2018;378:1976-86.

13. Bai R, Lv Z, Xu D, et al. Predictive biomarkers for cancer immunotherapy with immune checkpoint inhibitors. Biomark Res 2020;8:34.

14. Nishino M, Ramaiya NH, Hatabu H, et al. Monitoring immune-checkpoint blockade: response evaluation and biomarker development. Nat Rev Clin Oncol 2017;14:655-68.

15. Reuben A, Gittelman R, Gao J, et al. TCR Repertoire Intratumor Heterogeneity in Localized Lung Adenocarcinomas: An Association with Predicted Neoantigen Heterogeneity and Postsurgical Recurrence. Cancer Discov 2017;7:1088-97.

16. Chan TA, Yarchoan M, Jaffee E, et al. Development of tumor mutation burden as an immunotherapy biomarker: utility for the oncology clinic. Ann Oncol 2019;30:44-56.

17. Yu Y, Zeng D, Ou Q, et al. Association of Survival and Immune-Related Biomarkers With Immunotherapy in Patients With Non-Small Cell Lung Cancer: A Metaanalysis and Individual Patient-Level Analysis. JAMA 
Netw Open 2019;2:e196879.

18. Cheng F, Su L, Qian C. Circulating tumor DNA: a promising biomarker in the liquid biopsy of cancer. Oncotarget 2016;7:48832-41.

19. Sholl LM, Hirsch FR, Hwang D, et al. The Promises and Challenges of Tumor Mutation Burden as an Immunotherapy Biomarker: A Perspective from the International Association for the Study of Lung Cancer Pathology Committee. J Thorac Oncol 2020;15:1409-24.

20. Alexandrov LB, Nik-Zainal S, Wedge DC, et al. Signatures of mutational processes in human cancer. Nature 2013;500:415-21.

21. Devarakonda S, Rotolo F, Tsao MS, et al. Tumor Mutation Burden as a Biomarker in Resected Non-Small-Cell Lung Cancer. J Clin Oncol 2018;36:2995-3006.

22. Provencio M, Nadal E, Insa A, et al. Neoadjuvant chemotherapy and nivolumab in resectable non-small-cell lung cancer (NADIM): an open-label, multicentre, singlearm, phase 2 trial. Lancet Oncol 2020;21:1413-22.

23. Budczies J, Kazdal D, Allgäuer M, et al. Quantifying potential confounders of panel-based tumor mutational burden (TMB) measurement. Lung Cancer 2020;142:114-9.

24. Kazdal D, Endris V, Allgäuer M, et al. Spatial and Temporal Heterogeneity of Panel-Based Tumor Mutational Burden in Pulmonary Adenocarcinoma: Separating Biology From Technical Artifacts. J Thorac Oncol 2019;14:1935-47.

25. McGranahan N, Furness AJ, Rosenthal R, et al. Clonal neoantigens elicit $\mathrm{T}$ cell immunoreactivity and sensitivity to immune checkpoint blockade. Science 2016;351:1463-9.

26. Takamochi K, Takahashi F, Suehara Y, et al. DNA mismatch repair deficiency in surgically resected lung adenocarcinoma: Microsatellite instability analysis using the Promega panel. Lung Cancer 2017;110:26-31.

27. Zhao Z, Zhao D, Xia J, et al. Immunoscore Predicts Survival in Early-Stage Lung Adenocarcinoma Patients. Front Oncol 2020;10:691.

28. Bodor JN, Boumber Y, Borghaei H. Biomarkers for immune checkpoint inhibition in non-small cell lung cancer (NSCLC). Cancer 2020;126:260-70.

29. Brambilla E, Le Teuff G, Marguet S, et al. Prognostic Effect of Tumor Lymphocytic Infiltration in Resectable Non-Small-Cell Lung Cancer. J Clin Oncol 2016;34:1223-30.

30. Petersen RP, Campa MJ, Sperlazza J, et al. Tumor infiltrating Foxp3+ regulatory T-cells are associated with recurrence in pathologic stage I NSCLC patients. Cancer
2006;107:2866-72.

31. Budczies J, Kirchner M, Kluck K, et al. A gene expression signature associated with $\mathrm{B}$ cells predicts benefit from immune checkpoint blockade in lung adenocarcinoma. Oncoimmunology 2021;10:1860586.

32. Corredor G, Wang X, Zhou Y, et al. Spatial Architecture and Arrangement of Tumor-Infiltrating Lymphocytes for Predicting Likelihood of Recurrence in Early-Stage NonSmall Cell Lung Cancer. Clin Cancer Res 2019;25:1526-34.

33. Reuben A, Zhang J, Chiou SH, et al. Comprehensive T cell repertoire characterization of non-small cell lung cancer. Nat Commun 2020;11:603.

34. Oezkan F, He K, Owen D, et al. OA13.07 Neoadjuvant atezolizumab in resectable NSCLC patients: Immunophenotyping results from the Interim Analysis of the Multicenter Trial LCMC3. J Thorac Oncol 2019;14:S242-3.

35. Diem S, Schmid S, Krapf M, et al. Neutrophil-toLymphocyte ratio (NLR) and Platelet-to-Lymphocyte ratio (PLR) as prognostic markers in patients with nonsmall cell lung cancer (NSCLC) treated with nivolumab. Lung Cancer 2017;111:176-81.

36. Laza-Briviesca R, Cruz-Bermudez, A Casarrubios M. P2.04-10 Biomarkers of pathological response on neoadjuvant chemo-immunotherapy treatment for resectable stage IIIA NSCLC patients. J Thorac Oncol 2019;14:S711.

37. Forde P, Spicer J, Lu S. Nivolumab + platinum-doublet chemotherapy vs chemotherapy as neoadjuvant treatment for resectable (IB-IIIA) non-small cell lung cancer in the phase 3 CheckMate 816 trial. AACR Annual Meeting 2021. Abstract CT003. Presented April 10, 2021.

38. Lam V, Tran H, Vasquez M. MA23.02 Circulating tumor DNA analysis with a novel variant classifier for recurrence detection in resected, early-stage lung cancer. J Thorac Oncol 2018;13:S438.

39. Alipoor SD, Mortaz E, Varahram M, et al. The Potential Biomarkers and Immunological Effects of Tumor-Derived Exosomes in Lung Cancer. Front Immunol 2018;9:819.

40. Soung YH, Ford S, Zhang V, et al. Exosomes in Cancer Diagnostics. Cancers (Basel) 2017;9:8.

41. Gao S, Li N, Gao S, et al. Neoadjuvant PD-1 inhibitor (Sintilimab) in NSCLC. J Thorac Oncol 2020;15:816-26.

doi: 10.21037/asj-21-75

Cite this article as: Söyler Y, Y1lmaz Ü. Biomarkers under investigation for neoadjuvant immune checkpoint inhibitors in non-small cell lung cancer. AME Surg J 2022;2:37. 